\title{
Phosphonated superplasticizers synthesis: adsorption, dispersion and fluidification ability on calcium carbonate suspensions.
}

\author{
Tramaux A. ${ }^{1}$, Azéma N., ${ }^{1, *}$, David G. ${ }^{2}$, Negrell . $^{2}$, Poulesquen A. ${ }^{3}$, Haas J. ${ }^{3}$, Remond S. $^{4}$ \\ ${ }_{2}^{1}$ Centre des Matériaux de l'Ecole des Mines d'Ales, France. \\ 2 Laboratoire Ingénierie et Architecture Macromoléculaire, Institut Charles Gerhardt de Montpellier. \\ 3 Laboratoire de Physicochimie des Matériaux Cimentaires, Commissariat à l'Energie Atomique de Marcoule, Bagnols-sur-Cèze. \\ ${ }^{4}$ Mines Douai, LGCgE - GCE, F-59508 Douai, France.
}

\begin{abstract}
Superplasticizers are chemical admixtures used to fluidize cement pastes. Among them, polycarboxylate are comb-like copolymers with carboxylic acid and poly(ethylene glycol) side chains. We present here the synthesis of various polycarboxylate-like copolymers including phosphonic acid functions. Their adsorption, dispersion and fluidification efficiencies are evaluated on calcium carbonate suspensions, a mineral material commonly used to simulate early-age cement behaviour.
\end{abstract}

\section{Introduction}

Improving concrete fluidity is a matter of importance for concrete placing. More fluid the material is, easier the filling of the formwork. Although this high fluidity can be reached by increasing the water to cement ratio (W/C), an excess of water will lead to the weakening of long term mechanical properties. Moreover, due to drying shrinkage, cracks in material can occur. In order to avoid such inconvenient, concrete industry has developed admixtures named "superplasticizers"[1]. These admixtures can lower the viscosity of concrete without any change of the W/C ratio[2]. Since their invention in 1930, superplasticizers have become ones of the most used admixtures in the concrete industry and their use is almost systematic in today's concrete formulation. Using specific mixture proportions, it is even possible to prepare "self compacting concrete", that can be placed without any vibrations, under their own weight.

A major innovation in the superplasticizer field was in 1980 the development of polycarboxylate[3]. These admixtures were comb-like copolymers, petro-based, composed by a succession of methacrylic acid units in the main chain, and a substitution of a part of these methacrylic acid units by poly(ethylene glycol) methyl ether methacrylate, forming side chains from the main chain. Much more efficient than previous kinds of superplasticizers, polycarboxylate are still today the most used and also the most studied. Indeed, by changing structural parameters (DPn, molecular weight, side-chains ratio or length) it is possible to design various polycarboxylates, more or less efficient. Because of the wide variety of cement Portland compositions, the concrete industry has reported many issues of incompatibility between cement and admixtures, leading to a loss of efficiency of superplasticizers or other undesired effects[4]. One of the strongest interests of polycarboxylate is that their important structure diversity is useful to assure a good compatibility with cement compositions, even in presence of mineral additions (fly ash, slag, silica fume).

Origin of the fluidization action of polycarboxylate is decomposed in two steps: firstly, adsorption of the comb-like copolymer onto surface of the cement grain. Carboxylic acids are anionic functions fully deprotonated in alkali environment which have high affinity for positively charged surfaces, such as ettringite phases for example. Second step is the formation of a polymeric crown around particles, which generates steric hindrance and acts against the van der Walls attractions. Particles are thus dispersed, water trapped in agglomerates is released and very fine particles are generated. From a macroscopic point of view, the cement paste becomes more fluid and easier to place.

Even if polycarboxylate are widely used in building industry, various studies try to enhance their performances with structural modifications of macromolecular architecture, including the nature of anionic function. Some works tried to replace the carboxylic acid by phosphonic acid, with good results[5]. One of the interests for improving the affinity of the "anchor group" for mineral surfaces is the ionic competition resistance. A high ion concentration can drastically decrease superplasticizer adsorption efficiency and thus its fluidification effect. This occurs often in high sulfate content cement paste.

In this study we present the synthesis of various comblike copolymers, whose macromolecular structures are similar to standard polycarboxylate, but with phosphonic acid functions instead of carboxylic acid moieties. Adsorption, dispersion and fluidification efficiencies of these superplasticizers are evaluated on calcite suspensions, a model inert material widely used to simulate early-age cement behaviour. Finally, effect of

*Corresponding author: nathalie.azema@mines-ales.fr 
sulfate concentration is also investigated through addition of sodium sulfate.

\section{Materials and methods}

\subsection{Calcite}

Model mineral material chosen, calcite, is the most stable allotropes of calcium carbonate. It has been purchased from Omya company (ref: OMYA BL). This low soluble white powder is composed of monomorphic angular particles from 0.3 to $40 \mu \mathrm{m}$ diameter.

\subsection{Calcite suspensions preparation}

Initial suspensions were prepared mixing calcite, superplasticizer aqueous solution and the solution of interest (deionized water, limewater or limewater with sulfate ions) keeping constant the water/solid ratio $(\mathrm{W} / \mathrm{S}=0.5)$. The superplasticizer dosage corresponds to the mass of solid superplasticizer inserted over the mass of calcite. After at least 10 minutes of magnetic stirring, suspensions are diluted according to measurement requirements.

\subsection{Superplasticizers preparation}

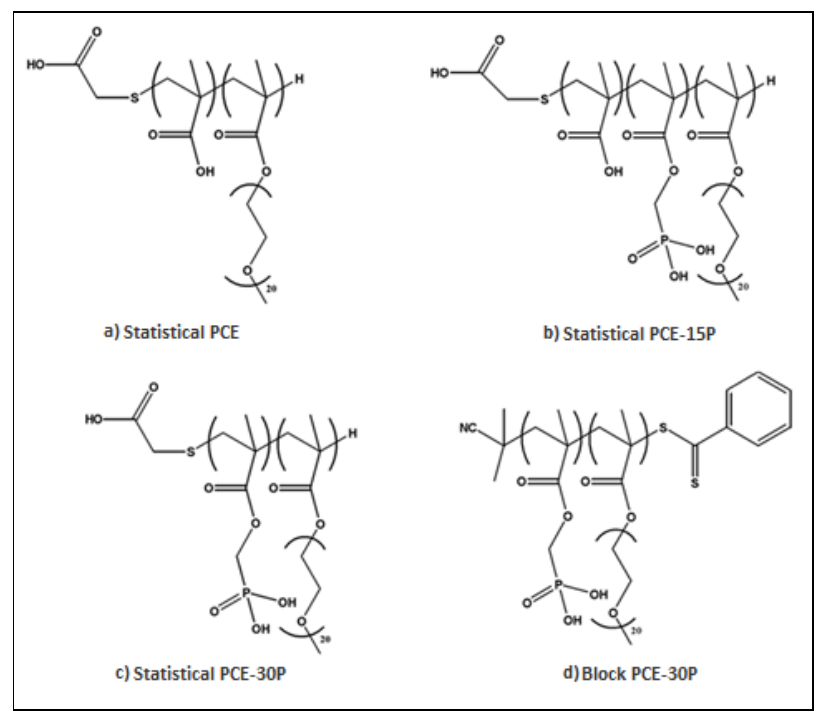

Fig. 1. Structures of synthetized copolymers

Four comb-like copolymers have been prepared according to two synthesis routes: three of them (a, b and c) are random copolymers and have been synthetized through conventional radical copolymerisation, using thioglycolic acid as chain transfer agent. The last one (d) is a block copolymer prepared by RAFT (Reversible Addition-Fragmentation Chain Transfer) copolymerisation, with CIDB as RAFT agent. These copolymers have been synthetized with the idea to keep constant macromolecular parameters such as DPn, side chains length and PEG side chains/anionic functions ratio. Once synthetized, these copolymers are conditioned in $100 \mathrm{~g} / \mathrm{L}$ aqueous solutions.

\subsection{Adsorption analysis}

Adsorption analyses were performed thanks to a Vario TOC cube analyser, from Elementar Company. After centrifugation of initial suspension, supernatant is filtered, diluted and then analysed. The polymer concentration is determined from integration of the area under the curve. Finally, thanks to the difference between amount of copolymer added and measured, the amount of copolymer adsorbed is expressed in $\mathrm{mg}$ per gram of calcite.

\subsection{Rheological measurements}

From a concentrated calcite suspension $(\mathrm{W} / \mathrm{S}=0.36)$ admixtured with $0.05 \%$ superplasticizer, rheological measurements were performed with an AR2000 Ex rheometer from TA instruments Company. The geometry employed was rotating vane. After a short preshear, suspension is sheared at $100 \mathrm{~s}^{-1}$ during 10 minutes. $\eta_{10}$ is the average viscosity of suspensions during these 10 minutes of analysis.

\subsection{Suspension stability evaluation}

Suspensions stability is investigated with a concentrated suspension light analyser, Turbiscan MA2000 from Formulaction Company. A 3 factor dilution with the liquid of interest $(\mathrm{W} / \mathrm{S}=3.5)$ is performed from initial calcite suspension before being inserted in a glass tube. Turbiscan analyses light transmitted and backscattered as a function of column height and time. Analyses last 30 minutes, with a scan every minute.

\section{Results and discussion}

\subsection{Macromolecular architectures description}

Statistical PCE is a comb-like copolymer whose structure corresponds to a standard polycarboxylate. Statistical PCE-30P has almost the same structure, except that all carboxylic acids are replaced by phosphonic acids. Statistical PCE-15P is intermediate, with half of anionic functions which are carboxylic acids and half are phosphonic acids. Block PCE-30P has a structure similar to statistical PCE-30P, but it is a block copolymer, expected to behave differently from the others. To our knowledge, it is the first time that a block copolymer is synthetized for superplasticizer applications[5].

Table 1. Copolymers structural parameters. * Mn and DPn are theoretical, according to reactant quantities employed.

\begin{tabular}{lcccccr}
\multicolumn{1}{c}{ Superplasticizer } & $\begin{array}{c}\text { Methacrylic } \\
\text { acid ratio }\end{array}$ & $\begin{array}{c}\text { Phosphonic } \\
\text { acid ratio }\end{array}$ & $\begin{array}{c}\text { PEG side chain } \\
\text { ratio }\end{array}$ & $\begin{array}{c}\text { PEG side } \\
\text { chain length }\end{array}$ & Mn* & DPn* \\
\hline Statistical PCE & $30 \%$ & - & $70 \%$ & 20 & $\approx 38500$ & 57 \\
Statistical PCE-15P & $15 \%$ & $15 \%$ & $70 \%$ & 20 & $\approx 35000$ & 50 \\
Statistical PCE-30P & - & $30 \%$ & $70 \%$ & 20 & $\approx 37000$ & 52 \\
Block PCE-30P & - & $30 \%$ & $70 \%$ & 20 & $\approx 38500$ & 52
\end{tabular}




\subsection{Superplasticizers adsorption capacity}

Suspensions were prepared with $0.5 \%$ superplasticizer dosage. Fig. 2 presents adsorption capacity of the four superplasticizers, in $\mathrm{mg}$ of copolymer adsorbed per grams of calcite.

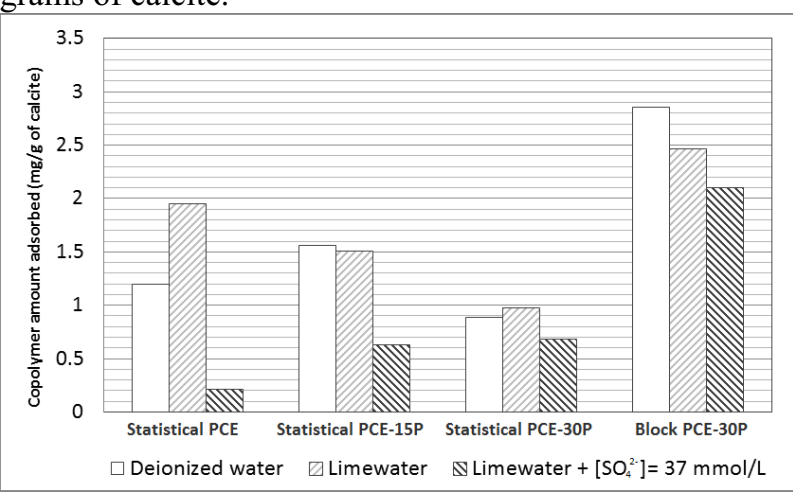

Fig. 2. Amount of copolymer adsorbed onto calcite in three different environments.

Using limewater is useful to get a $\mathrm{pH}$ of 12.8 , close to cement pore solution $\mathrm{pH}$. We can notice that few differences are visible between water and limewater. Only statistical PCE seems to adsorb in higher amount in limewater. This could be explained by the full deprotonation of $\mathrm{COOH}$ moieties in alkali environment, increasing affinity of the macromolecule for calcite. In all liquids studied, block copolymer provides much greater adsorption ability than statistical ones probably due to its different macromolecular structure close to surfactant ones. On the other hand, in water and in limewater adsorption ability of statistical copolymers seems to be less effective when carboxylic acid groups are substituted by phosphonic acid but not in presence of sulfate ions where the trend is reversed, due to the less sensitivity of phosphonic acid to ionic competition[6]. The more the macromolecule includes phosphonic acid, the less its adsorption is affected by sulfate ions. Moreover, even if the block PCE-30P seems to adsorb in higher proportions than others, we propose that not necessarily reveal higher affinity for mineral surfaces but may be due to another mode of adsorption of the "anionic block".

\subsection{Rheology measurements}

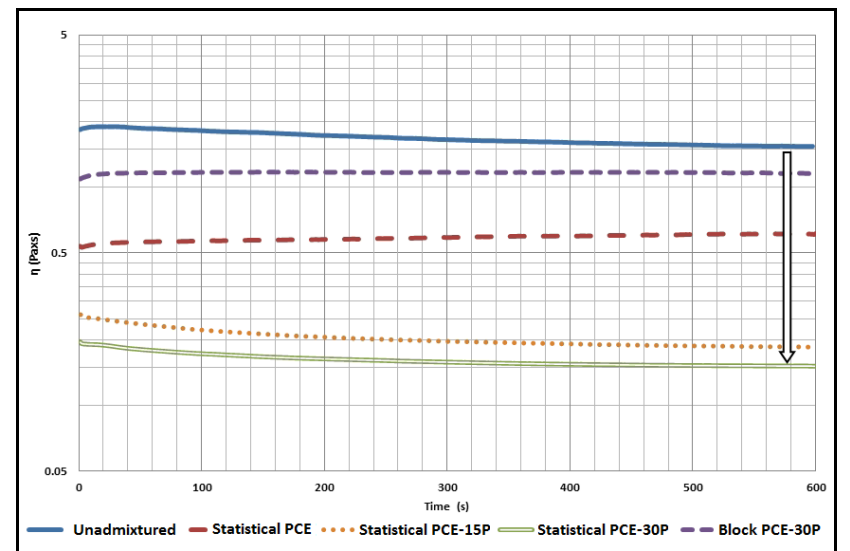

Fig. 3. Viscosity evolution of calcite suspensions admixtured with $0.05 \%$ of superplasticizer. Acquisitions in deionized water.
Fig.3 displays the viscosity of calcite suspension in deionized water when admixtured with $0.05 \%$ of superplasticizer. As a general trend, addition of superplasticizer decreases viscosity as substitution of carboxylic acid by phosphonic acid group, dividing the viscosity by a factor 10 for statistical PCE-30P. Despite the high adsorption ability of block PCE-30P, its adsorption in $\mathrm{CaCO}_{3}$ suspensions does not lead to a significant decrease of viscosity, as statistical copolymers.

Table 2 summarizes viscosities of suspensions in different solutions.

Table 2. Viscosities averaged over 600 seconds of calcite suspensions in three different environments.

\begin{tabular}{lccc} 
& \multicolumn{3}{c}{$\boldsymbol{\eta}_{\mathbf{1 0}}(\mathrm{Pa} \times \mathrm{s})$} \\
\cline { 2 - 4 } Copolymer & $\begin{array}{c}\text { Deionized } \\
\text { water }\end{array}$ & Limewater & $\begin{array}{c}\text { Limewater }+ \\
{\left[\mathrm{SO}_{4}{ }^{2}\right]} \\
=37 \mathrm{mmol} / \mathrm{L}\end{array}$ \\
\hline- & 1.68 & 1.52 & 2.54 \\
Stat. PCE & 0.58 & 0.35 & 2.51 \\
Stat. PCE-15P & 0.20 & 0.16 & 1.45 \\
Stat. PCE-30P & 0.16 & 0.13 & 0.35 \\
Block PCE-30P & 1.17 & 0.85 & 1.12
\end{tabular}

Same observations are made in all solutions studied, deionized water, limewater and limewater with sulfate ions. For water and limewater solutions, results seem to indicate that non adsorbed copolymer could have a role in particles dispersion[7] because viscosity decreases as well adsorption ability decreases. In presence of sulfate, all superplasticizers fluidizing efficiencies are lowered in agreement with lowest values of adsorption amounts. In particular statistical PCE, which doesn't contain phosphonic acid, undergoes drastic decrease of its efficiency. Its viscosity is almost the same than unadmixtured suspension.

\subsection{Calcite suspension stability}

Turbiscan MA 2000 allows the monitoring of transmitted and backscattered light intensity of calcite suspensions as a function of time. From the backscattered profile, we have developed a suspension stability index, named "A", taking into account all destabilizations phenomena. A is the "Area difference" of the two backscattered signal intensity curves, between $\mathrm{t}=6$ and $\mathrm{t}=0$ minutes. These two times have been chosen arbitrarily, to exhibit maximum differences between formulations, especially at intermediate dosages. When suspension is not stabilized, the signal evolves rapidly and the value of $\mathrm{A}$ is high. When suspension is stabilized, at high superplasticizer dosage, sedimentation is greatly slowed down, and the value of $\mathrm{A}$ is minimal. We consider that for a value below 50 area units, the suspension is stabilized in analysis time.

Fig.4. displays evolution of A according to amount of superplasticizer used. At high dosages, $(>0.2 \%)$ all copolymers lead to a stable suspension, except block copolymer until $0.5 \%$ for the same effect. Overall, block PCE-30P presents a lesser dispersion efficiency than others copolymers, in good agreement with rheological measurements. At low dosages (between 0.01 and $0.1 \%$ ), 

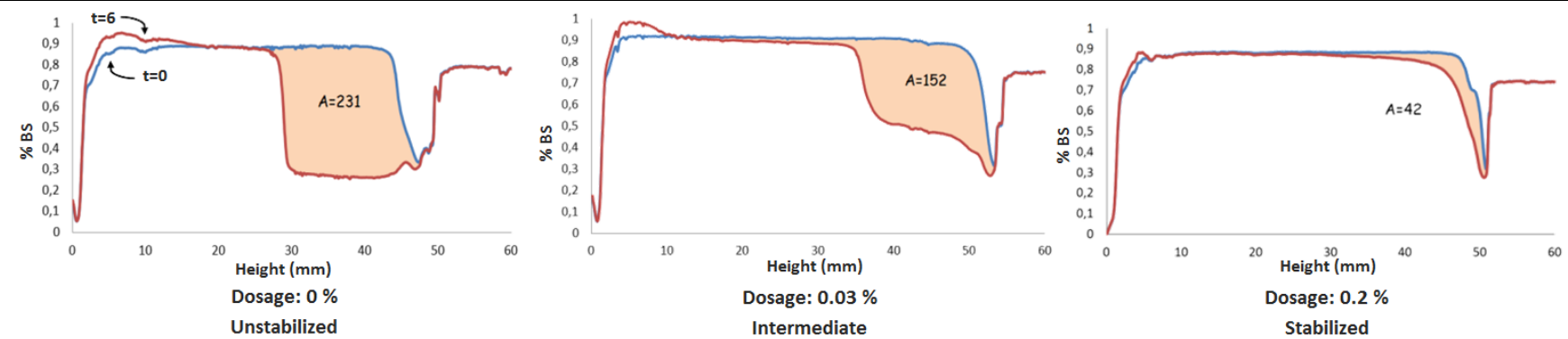

Fig. 5. Three examples of calculation of A with several dosages of statistical PCE in limewater. A is in area unit.

we can notice that the value of $\mathrm{A}$ is higher than in the case of unadmixtured suspension. This observation, may be probably explained by bridging phenomena[8]. Bridging is the multiple adsorption of one single macromolecule onto two or more particles at the same time when surfaces are not saturated. This phenomenon, well-known to occur at low polymer concentration, leads to formation of agglomerates, thus particles sedimentation.

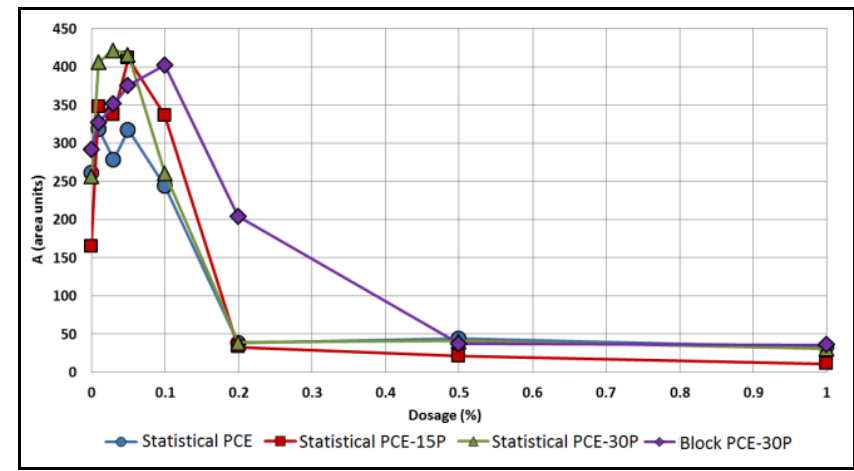

Fig. 4. Evolution of A parameter as a function of superplasticizer dosage. Acquisitions in deionized water.

Table 3 summarizes the amount of copolymer to use in order to get a value of A below 50 area units namely stabilized systems. As it was observed in adsorption analysis, few differences are noticeable between limewater and deionized water. Block copolymer have lowest dispersion ability in agreement with rheological results but an improvement seems to be observed in limewater. In presence of sulfate ions, statistical PCE30P keeps its efficiency whereas statistical PCE is no more able to lower A, which remains higher than 50 area units even with $1 \%$ dosage. Statistical PCE-15P, whose structure is intermediate between PCE and PCE-30P, display intermediate dispersion efficiency. These last results confirm that in presence of sulfate ions, superplasticizers have lowest fluidizing efficiencies.

Table 3. Superplasticizer dosage required (\%) to reach a value of $\mathrm{A}$ below 50 area units.

\begin{tabular}{lccc}
\hline \multicolumn{1}{c}{ Copolymer } & $\begin{array}{c}\text { Deionized } \\
\text { water }\end{array}$ & Limewater & $\begin{array}{c}\text { Limewater }+ \\
{\left[\mathrm{SO}_{4}^{2}{ }^{2}\right]} \\
=37 \mathrm{mmol} / \mathrm{L}\end{array}$ \\
\hline Stat. PCE & 0.2 & 0.2 & - \\
Stat. PCE-15P & 0.2 & 0.2 & 0.5 \\
Stat. PCE-30P & 0.2 & 0.2 & 0.2 \\
Block PCE-30P & 0.5 & 0.1 & 0.2
\end{tabular}

\section{Conclusion}

Four comb-like copolymers have been synthetized with different macromolecular architectures but with same molar mass, side chain length and anionic functions ratio. Their adsorption, dispersion and fluidification efficiencies have been investigated on calcite suspensions prepared in three different solutions: deionized water, limewater and limewater with sulfate ions. In water and limewater, few differences were observed between statistical copolymers. Only block copolymer leads to better adsorption ability but has in return the lowest effect on fluidization and dispersion of calcite suspensions. Although statistical copolymers present a lower adsorption ability, their dispersion and fluidization efficiency are more important probably due to non-adsorbed copolymer role.

Ionic competition leads to a lowest adsorption, dispersion and fluidification efficiencies of superplasticizers in presence of sulfate ions, however an increase of phosphonic acid group rate in statistical macromolecular structures make superplasticizers less sensitive to ionic competition.

\section{Acknowledgement}

This work was financed by LabEx CheMISyst of Balard Chemistry Center of Montpellier and ARMINES association (Paris), with a financial support from CEA of Marcoule (France).

\section{References}

[1] F. Winnefeld, S. Becker, J. Pakusch, and T. Götz, "Effects of the molecular architecture of comb-shaped superplasticizers on their performance in cementitious systems," Cem. Concr. Compos., vol. 29, pp. 251-262, 2007.

[2] D. Nagrockiene, I. Pundiene, and A. Kicaite, "The effect of cement type and plasticizer addition on concrete properties," Constr. Build. Mater., vol. 45, pp. 324-331, 2013.

[3] T. Hirata, A cement dispersant, in: JP Patent 84,2022, 1981, S59-018338.

[4] S. Hanehara and K. Yamada, "Interaction between cement and chemical admixture from the point of cement hydration, absorption behaviour of admixture, and paste rheology," Cem. Concr. Res., vol. 29, pp. 1159-1165, 1999.

[5] J. Plank, E. Sakai, C. W. Miao, C. Yu, and J. X. Hong, "Chemical admixtures - Chemistry, applications and their impact on concrete microstructure and durability," Cem. Concr. Res., vol. 78, pp. 81-99, 2015.

[6] F. Dalas, S. Pourchet, A. Nonat, D. Rinaldi, S. Sabio, and M. Mosquet, "Fluidizing efficiency of comb-like superplasticizers: The effect of the anionic function, the side chain length and the grafting degree," Cem. Concr. Res., vol. 71, pp. 115-123, 2015.

[7] A. Lange and J. Plank, "Contribution of non-adsorbing polymers to cement dispersion," Cem. Concr. Res., vol. 79, pp. 131-136, Jan. 2016.

[8] F. Lafuma, K. Wong, and B. Cabane, "Bridging of colloidal particles through adsorbed polymers," J. Colloid Interface Sci., vol. 143, no. 1, pp. 9-21, 1991. 